\title{
ATIVIDADES DE VIDA DIÁRIA COMO PREDITORES COGNITIVO EM IDOSOS INSTITUCIONALIZADOS
}

\author{
| Cristina Imaginário; ${ }^{1}$ Paulo Machado ${ }^{2}$; Magda Rocha ${ }^{3}$; Cristina Antunes ${ }^{4}$; Teresa Martins ${ }^{5}$ |
}

\section{RESUMO}

CONTEXTO: Partimos do pressuposto que a preservação do desempenho das atividades básicas e instrumentais de vida diária em idosos institucionalizados contribui para a potenciação e conservação da função cognitiva.

OBJETIVOS: Avaliar se a capacidade funcional para as atividades de vida diária prediz a função cognitiva dos idosos institucionalizados em Equipamentos Residenciais.

MÉTODOS: Estudo transversal correlacional preditivo, com recurso a amostra aleatória simples constituída por 475 sujeitos. Instrumentos: Mini-Mental State Examination (Guerreiro et al., 1994); Índice de Barthel (Araújo, Ribeiro, Oliveira e Pinto, 2007) e Escala de Lawton \& Brody (Araújo, Ribeiro, Oliveira, Pinto e Martins, 2008).

RESULTADOS: Os idosos que residem nas instituições residenciais são maioritariamente do género feminino, com idades compreendidas entre 65 e 104 anos $(M=83,94, \mathrm{DP}=7,21)$, na maioria viúvos e analfabetos. O modelo de regressão múltipla sugere que as atividades básicas e instrumentais de vida diária explicam cerca de $49 \%$ da variância existente do estado mental dos idosos. Verificou-se que as atividades instrumentais de vida diária correspondem ao preditor com maior peso, embora a contribuição das atividades básicas de vida diária seja também, estatisticamente, significativa para o modelo.

CONCLUSÕES: O modelo sugere que a manutenção da execução das atividades de vida diária beneficia o estado cognitivo nestes utentes. Através de intervenções de estimulação motora e social, os idosos poderão preservar a sua autonomia na realização de atividades de vida diária, que por sua vez potenciam uma maior saúde mental, melhor bem-estar e maior autoestima.

\section{PALAVRAS-CHAVE: Atividades básicas e instrumentais de vida diária; Estado cognitivo; Idosos institucionalizados}

\section{RESUMEN}

\section{"Actividades diarias como predictores del estado cognitivo en ancianos institucionalizados"}

CONTEXTO: Partimos del presupuesto que la preservación del desarrollo de actividades básicas e instrumentales de la vida diaria en ancianos institucionalizados contribuye para la mejoría y preservación de la función cognitiva.

OBJETIVOS: Evaluar si la capacidad funcional para las actividades de la vida diaria predice la función cognitiva de los ancianos institucionalizados en aparatos residenciales.

METODOLOGÍA: Una correlación predictiva con encuesta, usando muestra aleatoria simple de 475 sujetos. Instrumentos: Mini-Mental State Examination, (Guerrero et al., 1994) Índice de Barthel (Araujo, Ribeiro, Oliveira e Pinto, 2007) y la escala de Lawton \& Brody (Araújo, Ribeiro, Oliveira, Pinto e Martins, 2008).

RESULTADOS: Los ancianos que viven en instalaciones residenciales son en su mayoría mujeres, con edades comprendidas entre los 65 y 104 años $(\mathrm{M}=83.94, \mathrm{SD}=7.21)$, en su generalidad viudos $\mathrm{y}$ analfabetos. El modelo de regresión múltiple sugiere que las actividades básicas e instrumentales de la vida diaria explican aproximadamente el $49 \%$ de la variación existente del estado mental de las personas mayores. Se comprobó que las actividades instrumentales de la vida diaria corresponden al predictor con mayor peso, aunque la contribución de las actividades básicas de la vida diaria es, estadísticamente, significativa para el modelo.

CONCLUSIONES: El modelo sugiere que la manutención de ejecución continuada de las actividades diarias favorece el estado cognitivo en estos usuarios. A través de las intervenciones motora y social, las personas mayores pueden preservar su independencia en la realización de actividades de la vida diaria, que a su vez mejoran y potencializan una mejor y mayor salud mental, un mayor bien-estar y una mayor autoestima.

DESCRIPTORES: Actividades instrumentales y básicas de la vida diaria; Estado cognitivo; Ancianos institucionalizados

\section{ABSTRACT \\ "Activities of daily living as cognitive state predictors for the in- stitutionalized elderly"}

BACKGROUND: This study was based on the notion that preserving basic and instrumental activities of daily living can help the institutionalized elderly enhance and maintain their cognitive functions.

AIM: The aim of this study was to assess if the functional capacity for activities of daily living can predict cognitive functions of the institutionalized elderly in assisted living residences.

METHODS: A predictive transversal correlational study was carried out, using simple random sampling of 475 participants. Instruments: MiniMental State Examination (Guerreiro et al., 1994); Barthel's index (Araújo, Ribeiro, Oliveira and Pinto, 2007) and Lawton \& Brody's scale (Araújo, Ribeiro, Oliveira, Pinto and Martins, 2008).

RESULTS: The elderly in assisted living residences are primarily female, with ages ranging from 65 to $104(\mathrm{M}=83,94, \mathrm{DP}=7,21)$, most of them widowed and illiterate. The model of multiple regression suggests that basic and instrumental activities of daily living explain approximately $49 \%$ of the existing variance in the mental state of the elderly. The instrumental activities of daily living were shown to be the most significant predictor, though the contribution of basic activities of daily living is also statistically significant for the model.

CONCLUSIONS: The model suggests that these patients' cognitive state can benefit from maintaining their activities of daily living. The elderly might preserve their autonomy in carrying out activities of daily living through social and motor stimulation interventions, which in turn can improve mental health, well-being and self-esteem.

\section{KEYWORDS: Basic and instrumental activities of daily living; Cognitive state; Institutionalized elderly}

Submetido em 30-05-2017

Aceite em 09-11-2017

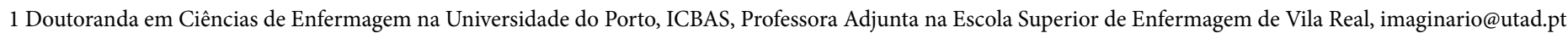
2 Professor Adjunto na Escola Superior de Enfermagem do Porto, 4200-072 Porto, Portugal, paulom@esenf.pt

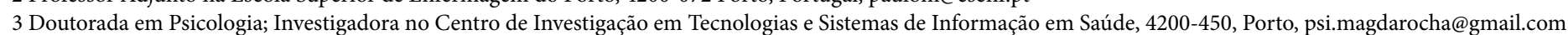
4 Professora Adjunta na Escola Superior de Enfermagem de Vila Real, 5000-801 Vila Real, Portugal, mantunes@utad.pt

5 Professora Coordenadora na Escola Superior de Enfermagem do Porto, 4200-072 Porto, Portugal, teresam@esenf.pt
}

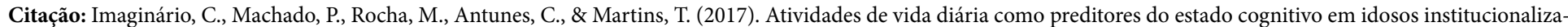
dos. Revista Portuguesa de Enfermagem de Saúde Mental (18), 37-43. doi: 10.19131/rpesm.0190 


\section{INTRODUÇÃO}

A avaliação da capacidade funcional é efetuada com base no desempenho das atividades de vida diária (AVD), sendo divididas em: atividades básicas de vida diária (ABVD), associadas ao autocuidado e à sobrevivência (como o alimentar-se, vestir-se, tomar banho, deambular, controlar esfíncteres); atividades instrumentais de vida diária (AIVD), que abrangem tarefas que permitem a manutenção da vida em comunidade (como realizar compras, utilizar o telefone, gerir assuntos económicos ou utilizar meios de transporte); e atividades avançadas de vida diária (AAVD), mais complexas, que se subdividem nos domínios físico, lazer, social e produtivo. Estas últimas exigem como expectável, maiores níveis de funcionalidade (Dias, Andrade, Duarte, Santos e Lebrão, 2015).

O declínio cognitivo pode associar-se a um padrão específico de perdas funcionais das AVD. Estas perdas de funcionalidade iniciam-se com um comprometimento nas AAVD, seguidas pelas AIVD e por último pelas ABVD (Dias et al., 2015). Os autores (Ibidem) reforçam a ideia de que a preservação do desempenho das AVD é um fator protetor na conservação das funções mentais. Assim, um estilo de vida ativo pode favorecer a proteção das funções cognitivas. Os idosos deverão ser incentivados a executar atividades quer instrumentais, quer intelectuais visando a promoção ou manutenção da capacidade cognitiva.

O processo de envelhecimento normativo é, na maioria das vezes, acompanhado de défices cognitivos. Deste modo, no idoso, a saúde mental tem uma importância primordial na prevenção do declínio cognitivo e do retardamento da dependência e da perda da capacidade de se autocuidarem (Apóstolo, Cardoso, Marta e Amaral, 2011). Há autores que defendem que a avaliação/ monitorização do estado cognitivo permite a deteção precoce de défices na funcionalidade, sendo por isso uma estratégia relevante na intervenção junto a populações idosas institucionalizadas (ou não) (Zimmermann, Leal, Zimmermann e Marques, 2015). Esta avaliação pode orientar para intervenções terapêuticas específicas, que de acordo com o estado inicial detetado, contribuem para a redução das perdas cognitivas (Mello, Haddad e Dellaroza, 2012; Zimmermmann et al.,2015). A literatura destaca que a estimulação cognitiva e atividade física são fundamentais na preservação da saúde dos idosos, particularmente dos institucionalizados (Loureiro, Lima, Silva e Najjar, 2011).
Assim, restrições na execução das ABVD e AIVD, nas atividades físicas e de lazer contribuem para o isolamento e limitam a interação social dos idosos, tornando-os mais dependentes, e concorrendo para a diminuição da capacidade funcional e cognitiva (Zimmermann, Leal, Zimmermann, Marques e Gomes, 2015). Com base no exposto Mello et al. (2012) defendem que a manutenção da atividade nos idosos quer no domínio mental, quer físico, permite o aumento da autonomia e promove uma melhor qualidade de vida. Os mesmos autores (Ibidem) referem ainda que a diminuição na realização das tarefas quotidianas desestimula a atividade mental, contribuindo para uma potencialização da institucionalização em Equipamentos Residenciais para Pessoas Idosas. Mais ainda, Bertoldi, Batista e Ruzanowsky (2015) referem que a alteração de ambiente, em concreto a institucionalização é uma das situações de risco para a potenciação do défice cognitivo, dado que o isolamento social conduz à perda da identidade, da liberdade, da autoestima e fatores conducentes à solidão, o que pode, por si só, justificar o declínio cognitivo.

Tendo em conta a associação entre capacidade cognitiva e funcionalidade, reforça-se a importância de intervenções no âmbito da reabilitação cognitiva, de modo a promover um melhor potencial de saúde. Reconhece-se que as atividades de estimulação cognitiva conduzem a uma melhoria na capacidade cognitiva (Loureiro et al., 2011).

Desta forma, a implementação de programas deste tipo é essencial para a saúde e para o bem-estar dos idosos institucionalizados, a fim de estimular a cognição, melhorar a autonomia e atenuar o impacto dos défices que surgem naturalmente nesta época do ciclo vital. As intervenções de estimulação cognitiva, se bem delineadas e estruturadas, constituem-se um coadjuvante elementar das terapêuticas farmacológicas (Costa e Sequeira, 2013).

O presente estudo tem por objetivo a resposta a duas questões de investigação:

1) Qual a percentagem de variância explicada no estado cognitivo dos idosos em estudo através dos preditores a) Atividades Básicas de Vida Diária e b) Atividades Instrumentais de Vida Diária;

2) Qual o preditor, entre os dois acima referidos com maior peso no critério estado cognitivo dos idosos institucionalizados em estudo. 


\section{MÉTODO}

\section{Desenho do Estudo}

Este é um estudo transversal que recorreu à estatística multivariada para tratamento dos dados. Trata-se de um desenho correlacional preditivo que recorreu à estatística paramétrica (regressão linear múltipla) em ordem a testar as questões de investigação formuladas à priori. A variável critério constitui-se enquanto o estado mental dos respondentes, sendo os preditores do modelo as AIVD e as ABVD.

\section{Amostra e Participantes}

Em abril de 2011 realizou-se a consulta à Carta Social (Ministério da Solidariedade e Segurança Social, 2011) com vista à identificação dos Equipamentos Residenciais para Pessoas Idosas (ERPI) existentes no distrito de Vila Real e em cada um dos seus conselhos, tendo sido identificados 56 ERPI com capacidade total para 2000 idosos. Estes ERPI apresentam-se, juridicamente, como Instituições Privadas de Solidariedade Social. À época encontravam-se institucionalizados 1913 utentes. Após a identificação dos ERPI existentes, efetuou-se um primeiro contacto (telefónico) com os responsáveis de cada uma das 56 instituições, cujo objetivo foi o agendamento de uma reunião de apresentação do projeto e dos responsáveis pelo mesmo. Deste contacto resultou a deslocação física do primeiro autor a cada instituição e respetiva apresentação do estudo a realizar. Por fim, foi efetivado o pedido de autorização, anexando ao mesmo o comprovativo da frequência do primeiro autor no curso de doutoramento em Ciências de Enfermagem no Instituto de Ciências Biomédicas Abel Salazar e ainda, a declaração do consentimento livre e informado a utilizar no estudo. Só foram constituídas intervenientes nesta investigação as instituições que outorgaram a sua autorização escrita à sua participação e destas, aquando da colheita de dados, as que expuseram disponibilidade na ocasião para integrarem a amostra. No momento imediatamente anterior à recolha de dados, foi dado conhecimento aos responsáveis de cada instituição do protocolo a utilizar na recolha de dados. A colheita dos dados foi efetivada no período que mediou os meses de agosto de 2014 e julho de 2015.

Do ponto de vista da recolha geográfica da amostra, trata-se de uma amostra de conveniência, porém que tomou em linha de conta, dentro de cada instituição participante no estudo, a aleatoriedade da recolha de dados.
Assim a amostra descreve-se como não representativa do ponto de vista geográfico, embora tenha sido projetada como probabilística em cada instituição. A recolha foi levada a cabo de acordo com a colheita aleatória simples, com a atribuição de um número relativo ao nome de cada utente dentro de um saco, sendo retirados posteriormente, ao acaso, $30 \%$ dos sujeitos residentes em cada instituição. A percentagem foi restituída sempre que não existia aceitação por parte do respondente em participar no estudo.

Os participantes são oriundos na sua totalidade do interior norte de Portugal, estando distribuídos por 25 ERPI, aqueles que concordaram participar no estudo. Esta amostra é formada por 475 respondentes, de ambos os géneros (nmasculino $=152$; nfeminino $=323$ ), apresentando idades entre os 65 e 104 anos $(\mathrm{M}=83,94$, $\mathrm{DP}=7,21)$. Quanto ao estado civil, $16,00 \%(\mathrm{n}=76)$ são casados ou encontram-se em situação de união de facto, $21,50 \%(\mathrm{n}=102)$ são solteiros, sendo a amostra maioritariamente $60,00 \%(n=285)$ constituída por indivíduos viúvos. Ainda ao nível do estado civil, a amostra contou com a participação de 2,50\% $(n=12)$ idosos divorciados. A grande maioria dos participantes são analfabetos $53,60 \%(n=254)$ representando mais de metade do efetivo. Seguem-se-lhe em termos de escolaridade, aqueles que completaram 4 anos do $1^{\circ}$ ciclo do ensino básico $22,80 \%$ ( $n=108)$, em seguida o grupo que completou 3 anos do $1^{\circ}$ ciclo do ensino básico $17,50 \%(n=83)$. Os restantes participantes distribuem-se em termos de escolaridade com um ano $1,70 \%(\mathrm{n}=8)$, dois anos $1,50 \%$ $(\mathrm{n}=7)$, cinco ou nove anos $0,40 \%(\mathrm{n}=2)$, seis anos $0,80 \%(n=4)$, sete, oito ou 10 anos $0,20 \%(n=1)$ e, apenas 3 entre eles com 11 anos de escolaridade $(0,60 \%)$.

\section{Materiais e Procedimentos}

Em ordem a avaliar o grau de independência para a realização das ABVD recorreu-se ao Índice de Barthel, foi desenvolvido por Mahoney \& Barthel em 1965. O instrumento foi posteriormente validado para a população portuguesa por Araújo, Ribeiro, Oliveira e Pinto (2007). De acordo com os autores originais e com a versão portuguesa do instrumento (Ibidem), este faculta informação pertinente, não só a partir das pontuações totais, mas também a partir das classificações parciais, permitindo deste modo, observar quer as incapacidades específicas, quer o ajustamento dos cuidados às necessidades individuais de cada utente. $\mathrm{O}$ índice tem demonstrado uma elevada consistência interna ( $\alpha$ $=0,96$ ) (Araújo et al., 2007). 
A funcionalidade relativa às AIVD foi avaliada com recurso à Escala de Lawton \& Brody (1969). O instrumento foi validado para a população portuguesa por Araújo, Ribeiro, Oliveira, Pinto e Martins (2008). A escala apresenta um nível de fidelidade elevado, avaliado através do alfa de Cronbach $(\alpha=0,94)$. Araújo et al. (2008) indica tratar-se de uma medida genérica que avalia o nível de dependência da pessoa idosa na realização das suas AIVD. A pontuação atribuída varia entre 0 (máxima dependência) e os 23 pontos (independência total).

Quanto à avaliação do estado mental dos respondentes, foi utilizado o Mini-Mental State Examination (MMSE), desenvolvido por Folstein, Folstein \& McHugh (1975), cuja adaptação sistemática e transcultural para a população portuguesa foi efectuada por Guerreiro et al. (1994). O MMSE está amplamente creditado como sendo o instrumento mais adequado para a avaliação cognitiva breve de populações com baixa escolaridade, em idades avançadas. Estudos internacionais, transversais e longitudinais, referenciados por vários autores portugueses (Morgado, Rocha, Maruta, Guerreiro e Martins, 2009), estabeleceram valores normativos ajustados à idade e/ou à escolaridade dos sujeitos submetidos a avaliação. O MMSE é constituído por 30 questões, sendo atribuído um ponto por cada resposta correta, num total de 30 pontos. Os valores de corte para detecção de défice cognitivos propostos por Morgado et al. (2009) foram: 22 pontos para sujeitos com escolaridade entre zero e os dois anos; 24 pontos com literacia entre três e seis anos e 27 pontos para sujeitos com escolaridade superior a sete anos.

Finalmente foi utilizado um questionário de recolha de dados sociodemográficos, construído especificamente para este estudo, de modo a obter os dados que permitem a descrição aturada da amostra em estudo.

Ao nível dos procedimentos, todos os dados do protocolo foram recolhidos pela primeira autora. Foram salvaguardados os princípios éticos e os direitos do anonimato, de sigilo e de confidencialidade dos dados (ao nível da recolha e do tratamento dos mesmos). $\mathrm{O}$ procedimento relativo ao consentimento livre e informado por parte dos idosos foi levado a cabo tendo em conta os preceitos éticos da investigação em ciências humanas, observando a transferência da informação referente ao investigador, à natureza da investigação e aos objetivos da mesma. Devido às limitações etárias e de escolaridade dos participantes, os questionários foram aplicados oralmente.
O projeto foi aprovado pela comissão de ética do Instituto de Ciências Biomédicas Abel Salazar com número de registo 166/2016.

\section{Estatísticas e Análises dos Dados}

$\mathrm{Na}$ análise de dados utilizou-se a regressão linear múltipla, com recurso ao programa IBM $^{\circledast}$ SPSS $^{\circledast}$ (versão 22). A magnitude do efeito à posteriori, foi calculada com recurso ao f de Cohen (f2Cohen)), utilizando a calculadora online de Soper (2016).

\section{RESULTADOS}

\section{Análises Preliminares}

Foram testados os pressupostos relativos à utilização da regressão linear múltipla, tendo-se verificado a existência de correlações positivas e significativas entre as variáveis preditoras e a variável critério. Assim a correlação entre o score total do MMSE e o somatório do Índice de Barthel é positivo e moderado $(r=0,57, \mathrm{p} \leq$ 0,01 ), o mesmo sucedendo no resultado relativo à correlação de Pearson entre a variável critério e o preditor constituído pelo score da Escala de Lawton \& Brody ( $\mathrm{r}$ $=0,65, \mathrm{p} \leq 0,01)$. Estes valores indicam uma associação média entre variáveis, dando conta, como esperado, da não existência de multicolinariedade (Tabachnick \& Fidell, 2013). Também o valor da tolerância da colinariedade obtido $(0,58)$ confirma que não há violação deste pressuposto. Em termos de normalidade, a análise do gráfico P-P normal dos resíduos da regressão estandardizada observa os pontos da distribuição como contíguos à reta de regressão hipotética. Mais ainda, o gráfico de dispersão dá conta de uma distribuição onde a maior parte dos resíduos do score estão no centro, reforçando mais uma vez o cariz normativo da distribuição em estudo. Foram ainda observados os outliers tendo em conta a existência dos preditores e o respetivo valor crítico de avaliação dos valores da distância Mahalanobis para um valor de significância de $\mathrm{p} \leq 0,01$. $\mathrm{O}$ valor critério obtido foi de 13,82 . Tendo em conta a observação dos valores extremos verificou-se a existência de três outliers, optando-se pela decisão teóricoempírica da sua não retirada considerando o tamanho da $\operatorname{amostra}(\mathrm{N}=475)$.

\section{Regressão Linear Múltipla}

A análise do sumário do modelo da regressão linear múltipla, cujos preditores do Estado Mental foram, respetivamente, os scores obtidos nas ABVD e ainda a funcionalidade AIVD, indicou que este modelo explica cerca de $49 \%$ da variância existente no Estado Mental dos idosos em estudo $(\mathrm{R} 2=0,49)$. 
Tendo em conta o resultado obtido na Análise Univariada de Variância (ANOVA) verificou-se que este resultado é estatisticamente significativo $[\mathrm{F}(2,474)=$ $223,89, \mathrm{p} \leq 0,01]$, sendo o tamanho do efeito, ou a magnitude da associação entre preditores e critério, considerado grande ( $f 2$ Cohen $=0,96$ ) de acordo com Cohen (como citado em Lindenau e Guimarães, 2012).

Considerando a segunda questão de investigação, verificou-se que o preditor com maior peso no modelo são os scores obtidos a partir da Funcionalidade relativa às AIVD, representadas pelo score total da Escala de Lawton \& Brody, pese embora a contribuição das ABVD, avaliada através do Índice de Barthel seja igualmente estatisticamente significativa para o modelo. A Tabela 1 sumariza estes resultados.

Tabela 1 - Sumário da Regressão Linear Múltipla para as variáveis Preditoras do Estado Cognitivo em Idosos

\begin{tabular}{|l|c|c|c|c|c|}
\hline \multirow{2}{*}{} & \multicolumn{5}{|c|}{ Variável Critério (Estado Mental1) } \\
\cline { 2 - 6 } & $\begin{array}{l}\text { Coeficientes não } \\
\text { estandardizados }\end{array}$ & $\begin{array}{c}\text { Coeficientes } \\
\text { estandardizados }\end{array}$ & \multicolumn{2}{|c|}{ Teste $\mathrm{t}$} \\
\hline Variáveis & $\mathrm{B}$ & $\mathrm{EP}$ & $\beta$ & $\mathrm{t}$ & $\mathrm{p}$ \\
\hline ABVD & 0,23 & 0,05 & 0,22 & 5,09 & 0,00 \\
\hline AIVD & 0,62 & 0,05 & 0,54 & 12,41 & 0,00 \\
\hline R2 & & 0,49 & & & \\
\hline F & & $223,89^{\star *}$ & & & \\
\hline
\end{tabular}

Nota. 1 = Estado Mental tal como avaliado através do score total do Mini Mental State Examination, adaptação à população portuguesa de Guerreiro et al. (1994); $\mathrm{ABVD}=$ Atividades Básicas de Vida Diária; AIVD = Atividades Instrumentais de Vida Diária. ${ }^{\star} \mathrm{p} \leq .05 ;{ }^{* *} \mathrm{p} \leq .01 ; \mathrm{B}=$ Valor de Beta não estandardizado; $\mathrm{EP}=$ Erro Padrão; $\beta=$ Valor Beta Estandardizado; $t=$ valor do Teste $t, p=$ significância do Teste t.

\section{DISCUSSÃO}

O modelo explicativo mostra que, a capacidade funcional para as ABVD e as AIVD explicam $49 \%$ da variância existente do estado mental dos idosos, ou seja, um valor bastante expressivo. Daqui resulta a importância de se promover programas que exercitem as ABVD e AIVD, de acordo com uma cuidada avaliação de necessidades de cada pessoa. Poderemos também equacionar que se o idoso mostrar uma baixa adesão a atividades de estimulação cognitiva, ou por outro lado, não haja grande oferta destas atividades, poderemos recorrer a atividades instrumentais, de lazer ou sociais com vista à promoção/preservação da capacidade cognitiva.

Contudo, programas que alinhem o desenvolvimento de atividades de estimulação cognitiva e programas de treino de exercício e participação social serão ainda mais potenciadores de uma vida com maior significado e qualidade de vida.
Sendo indiciado pela literatura que existem múltiplos fatores de ordem física, psicológica e social que podem afetar o estado cognitivo (Pereira, Roncom e Carvalho, 2011), a execução das ABVD e AIVD, em particular das últimas, porque de complexidade mais elevada parecem, assim, promover a estimulação mental, constituindo-se como fator protetor da estabilidade das funções cognitivas.

Quando analisamos o peso de cada um dos tipos de AVD, para explicar um bom desempenho cognitivo, verificamos que a capacidade funcional para realizar AIVD tem maior peso como preditor no modelo, que a capacidade para realizar ABVD. A realização de AIVD favorece a exercitação das funções mentais, já que colocam o indivíduo perante situações cuja resolução exige um maior nível de complexidade que as ABVD. Zimmermann et al. (2015) referem que a restrição na execução de AIVD ou de lazer contribui para a diminuição das capacidades cognitivas. Deste modo, os estímulos intelectuais, recreativos e sociais, convergem para a manutenção mais saudável das funções cognitivas.

Perante estes resultados considera-se que nos ERPI devam existir equipas multidisciplinares com formação e competências necessárias à estimulação cognitiva e de AIVD. Esta equipa deverá incluir obrigatoriamente o profissional de enfermagem, já que este profissional mostra reunir um conjunto de competências alargadas, quer no planeamento e execução dessas atividades, quer na avaliação e monitorização dos resultados obtidos. O enfermeiro é detentor de competências que lhe permitem identificar e implementar intervenções no âmbito da promoção da autonomia, vigilância da saúde, reabilitação, prevenção de eventuais dificuldades clínicas, supervisão de atividades e estimulação para a execução das AVD e atividades interpessoais e socioculturais (Aleixo, Escoval, Fontes e Fonseca, 2011). Assim a presença de profissionais de enfermagem nessas instituições contribui para a diminuição do agravamento do estado de saúde dos idosos bem como no declínio da funcionalidade ao nível das AVD (Aleixo et al., 2011). É necessário mostrar aos responsáveis pelos ERPI e ao poder político que o investimento em cuidados de promoção e manutenção do potencial de saúde traduz ganhos em saúde, nomeadamente na autonomia e funcionalidade dos idosos. 


\section{CONCLUSÕES}

Face aos resultados deste estudo verificamos que a inexistência ou privação de atividades intelectuais e físicas sugerem a indução de declínios cognitivos. Reconhecese que a manutenção de atividades estimulantes ao nível mental e físico beneficiam a preservação da função cognitiva. Sendo assim, consideramos que o proporcionar de atividades de estimulação da memória, cálculo, memorização visual, leitura (ou equivalente) entre outras, devam estar disponíveis com o desenvolvimento crescente nos estabelecimentos residenciais para idosos. A este propósito, e reforçando o que acabou de ser dito, Loureiro et al. (2011) afirmam que, para a reabilitação cognitiva eficaz devem ser utilizadas atividades que estimulem a manutenção das atividades de concentração, a sequência de pensamento, a atenção e a capacidade de autonomia.

Apesar dos resultados explicativos do modelo serem robustos, uma das limitações do estudo decorre da ausência de outras variáveis explicativas concorrentes no bloco dos preditores. O padrão nutricional, o sedentarismo, o perfil de autocuidado, ou mesmo variáveis psicossociais poderão complementar os preditores do estado cognitivo.

Quanto a pistas para futuras investigações seria interessante realizar estudos comparativos entre idosos a participar em programas de estimulação de AIVD e ABVD, e comparar o desempenho cognitivo com um grupo de idosos sem esse tipo de estimulação. Estes estudos deverão ter em conta a avaliação prévia das linhas basais de funcionamento cognitivo, podendo assim observar-se até que ponto a eficácia destas intervenções se verifica.

Por outro lado, as avaliações e a promoção, de programas de estimulação de AIVD e ABVD à medida, implicam a existência de equipas multidisciplinares nos equipamentos residenciais, equipas essas que não podem prescindir do enfermeiro, quer enquanto promotor destas ações, quer enquanto formador dos membros das equipas que estão mais continuamente no terreno, caso dos auxiliares dos equipamentos residenciais. Também a implementação destes programas em contexto comunitário será uma mais-valia, na medida que promovem não só o bem-estar dos idosos, mas também, mantêm por mais tempo a autonomia e a atividade dos idosos, retardando a sua institucionalização. A estas questões não estará alheia a manutenção das redes interpessoais significativas, ou seja, uma maior proximidade de laços afetivos com filhos, cônjuge e demais família alargada.
Urge assim a criação de políticas de incentivo e proteção ao envelhecimento ativo, num país que como sabemos se apresenta demasiadamente envelhecido.

\section{REFERÊNCIAS BIBLIOGRÁFICAS}

Aleixo, T., Escovala, A., Fontes, R., e Fonseca, C. (2011). Indicadores de qualidade sensíveis aos cuidados de enfermagem em lares de idosos. Revista de Enfermagem Referência, serIII(3),141-149.

Apóstolo, J. L. A., Cardoso, D. F. B., Marta, L. M. G., e Amaral, T. I. O. (2011). Efeito da estimulação cognitiva em idosos. Revista de Enfermagem Referência, III série(5), 193-201.

Araújo, F., Ribeiro, J. L. P., Oliveira. A., Pinto, C., e Martins, T. (2008). Validação da escala de Lawton e Brody numa amostra de idosos não institucionalizados. In I. Leal, J. L. P. Ribeiro, I. Silva, e S. Marques (Eds.), Actas do $7^{\circ}$ congresso nacional de psicologia da saúde (pp. 217-220). Lisboa: ISPA.

Araújo, F., Ribeiro, J. L. P., Oliveira, A., e Pinto, C. (2007). Validação do Índice de Barthel numa amostra de idosos não institucionalizados. Revista Portuguesa de Saúde Pública, 25(2), 59-66.

Bertoldi, J. T., Batista, A. C., e Ruzanowsky, S. (2015). Declínio cognitivo em idosos institucionalizados: Revisão da literatura. Cinergis, 16(2), 152-156.

Costa, A. R. D., e Sequeira, C. (2013). Efetividade de um programa de estimulação cognitiva em idosos com défice cognitivo ligeiro. Revista Portuguesa de Enfermagem de Saúde Mental (3), 14-20.

Dias, E. G., Andrade, F. B., Duarte, Y. A. O., Santos, J. L. F., e Lebrão, M. L. (2015). Atividades avançadas de vida diária e incidência de declínio cognitivo em idosos: Estudo SABE. Cadernos de Saúde Pública, 31(8), 1623-1635.

Folstein, M., Folstein, S., \& McHugh, P. (1975). MiniMental State: A practical method for granding the cognitive state of patients for the clinician. Journal of Psychiatric Research, 12(3), 189-198. 
Guerreiro, M., Silva, A. P., Botelho, M., Leitão, O., Castro-Caldas, A., e Garcia, C. (1994). Adaptação à população portuguesa da tradução do Mini-Mental State Examination (MMSE). Revista Portuguesa de Neurologia, 1, 9-10.

Lawton, M. P., \& Brody, M. H. (1969). Assessment of older people: Self-maintaining and instrumental activities of daily living. The Gerontologist, 9(3), 179-186.

Lindenau, J. D. R., e Guimarães, L. S. P. (2012). Calculando o tamanho do efeito no SPSS. Revista HCPA, 32(3), 363-381.

Loureiro, A. P. L., Lima, A. A., Silva, R. C. G., e Najjar, E. C. A. (2011). Reabilitação cognitiva em idosos institucionalizados: Um estudo piloto. Revista de Terapia Ocupacional, 22(2), 136-144.

Mahoney, F. I., \& Barthel, D. W. (1965). Functional evaluation: The Barthel Index. Maryland State Medical Journal, 14, 61-65.

Mello, B. L. D., Haddad, M. C. L., e Dellaroza, M. S. G. (2012). Avaliação cognitiva de idosos institucionalizados. Acta Scientiarum. Health Sciences, 34(1), 95-102.

Ministério da Solidariedade e Segurança Social. (2011). Carta social: Rede de serviços e equipamentos: Relatório 2011. Lisboa: Ministério da Solidariedade e Segurança Social.
Morgado, J., Rocha, C. S., Maruta, C., Guerreiro, M., e Martins, I. P. (2009). Novos valores normativos do Mini-Mental State Examination. Sinapse, 2(9), 10-16.

Pereira, M. G., Roncom, J., e Carvalho, H. (2011). Aspetos psicológicos e familiares do envelhecimento. In $\mathrm{Z}$. Azeredo, O idoso como um todo (pp. 101-130). Viseu: PsicoSoma.

Soper, D. S. (2016). Effect size calculator for multiple regression [Software]. Disponível em http://www.danielsoper.com/statcalc

Tabachnick, B. G., \& Fidell L. S. (2013). Using multivariate statistics ( $6^{\mathrm{a}}$ ed.). Boston: Pearson.

Zimmermann, I. M. M., Leal, M. C. C., Zimmermann, R. D., Marques, A. P. O., e Gomes, E. C. C. (2015). Fatores associados ao comprometimento cognitivo em idosos institucionalizados: Revisão integrativa. Revista de enfermagem UFPE on line, 9(12), 1320-1328.

Zimmermann, I. M. M., Leal, M. C. C., Zimmermann, R. D., e Marques, A. P. O. (2015). Idosos institucionalizados: Comprometimento cognitivo e fatores associados. Geriatrics, Gerontology and Aging, 9(3), 86-92.

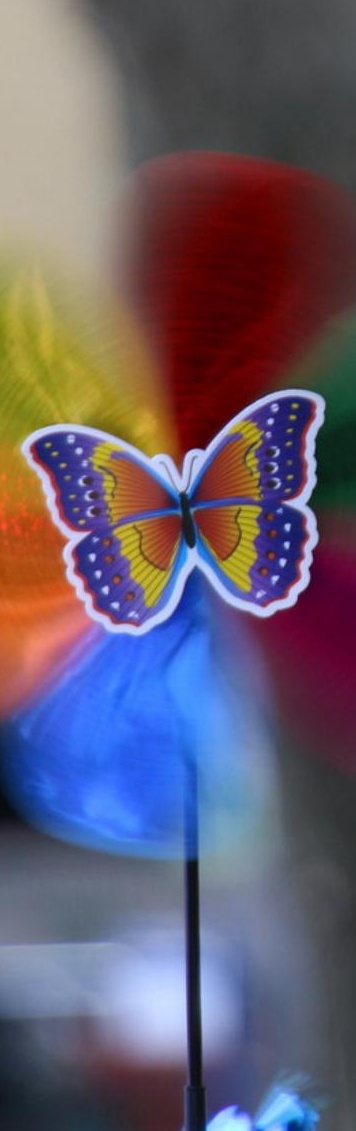

